\title{
Magnetically controlled negative refraction of solitons in liquid crystals
}

Vladlen G. Shvedov, Yana V. Izdebskaya, Yan Sheng, and Wieslaw Krolikowski

Citation: Appl. Phys. Lett. 110, 091107 (2017); doi: 10.1063/1.4977432

View online: http://dx.doi.org/10.1063/1.4977432

View Table of Contents: http://aip.scitation.org/toc/apl/110/9

Published by the American Institute of Physics

\section{Articles you may be interested in}

Complementary split-ring resonator antenna coupled quantum dot infrared photodetector

Appl. Phys. Lett. 110, 091106091106 (2017); 10.1063/1.4977427

Optofluidic Fano resonance photonic crystal refractometric sensors

Appl. Phys. Lett. 110, 091105091105 (2017); 10.1063/1.4977563

Thermal behavior and carrier injection of $\mathrm{GaAs} / \mathrm{GaP}$ quantum dots light emitting diodes

Appl. Phys. Lett. 110, 091101091101 (2017); 10.1063/1.4977716

Electrically tunable all-dielectric optical metasurfaces based on liquid crystals

Appl. Phys. Lett. 110, 071109071109 (2017); 10.1063/1.4976504

Direct observation of resonance scattering patterns in single silicon nanoparticles

Appl. Phys. Lett. 110, 091108091108 (2017); 10.1063/1.4977570

Surface effects on the photoconducting properties of $\mathrm{SrTiO} 3$ thin films

Appl. Phys. Lett. 110, 091103091103 (2017); 10.1063/1.4976944

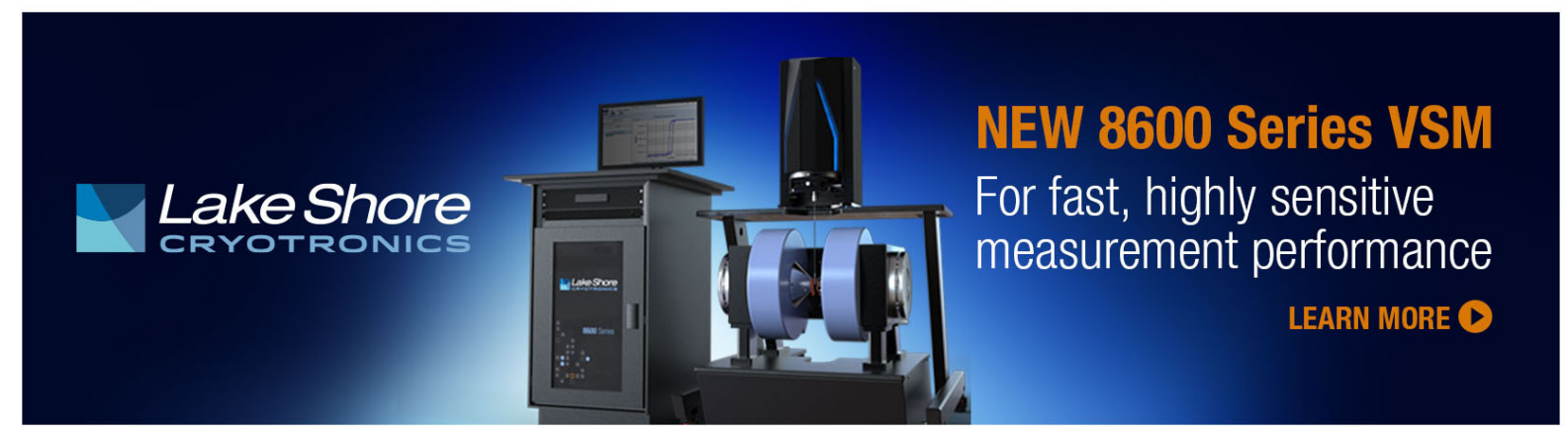




\title{
Magnetically controlled negative refraction of solitons in liquid crystals
}

\author{
Vladlen G. Shvedov, ${ }^{1}$ Yana V. Izdebskaya, ${ }^{1, a)}$ Yan Sheng, ${ }^{1}$ and Wieslaw Krolikowski ${ }^{1,2}$ \\ ${ }^{1}$ Laser Physics Center, Research School of Physics and Engineering, Australian National University, \\ Canberra, ACT 0200, Australia \\ ${ }^{2}$ Science Program, Texas A\&M University at Qatar, Doha, Qatar
}

(Received 2 January 2017; accepted 13 February 2017; published online 1 March 2017)

\begin{abstract}
We demonstrate magnetically controlled refraction of a spatial soliton forming beam at the interface between an isotropic material and a uniaxial nematic liquid crystal, in which the optical axis together with an incident beam can make any desired angles with the interface. Depending on the direction of the applied magnetic field and incident beam angle, the refracted soliton forming beam experiences either positive or negative refraction. Published by AIP Publishing.

[http://dx.doi.org/10.1063/1.4977432]
\end{abstract}

Refraction of light at the interface between transparent media is one of the most common optical phenomena used to steer and guide light in photonics devices. In isotropic lossless media, the light energy fluxes (the Poynting vector) of the incident and refracted beams always lie on opposite sides of the normal to the interface separating the media, and hence, light undergoes standard (positive) refraction (PR). In general, however, refraction of light can take an anomalous character with the incident and refracted energy fluxes propagating on the same side of the interface normal. ${ }^{1}$ This situation represents so-called negative refraction (NR) and can occur when light waves travel at an oblique angle through an interface of absorptive media, ${ }^{1}$ metamaterials, ${ }^{2,3}$ or anisotropic media. ${ }^{4-14}$ This situation involves both photonic crystals ${ }^{13,14}$ and natural or artificial birefringent materials. ${ }^{4-12}$

The negative refraction in the birefringent materials stems from the walk-off between the wave-vector and the Poynting vector in propagation of an extraordinary-polarized wave. ${ }^{4}$ This type of NR has been recently demonstrated in solids-positive uniaxial crystals ${ }^{4-7}$ and in anisotropic soft matter, such as nematic liquid crystals (NLCs) ${ }^{8-11}$ Refractions in the uniaxial crystals are governed by the relationship between the incidence angle of the light beam and the fixed orientation of the optical axis of the crystal and therefore can be varied only by the change of the incidence angle. This restricts the possible angular range where the NR can be observed.

In contrast, the positional flexibility of the rodlike molecules of NLCs allows for an easy alignment of their orientation and hence tuning the optical axis of NLCs by external factors such as stress, electric, or magnetic fields. ${ }^{15}$ Thanks to this feature, the NR has been observed for freepropagating laser beams in a planar NLC geometry with externally applied electric field ${ }^{8}$ and in a NLC prism in the presence of the external magnetic field used to reorient the molecular director in one certain plane. ${ }^{10} \mathrm{NR}$ has also been shown for self-confined beams ${ }^{9}$ in a thin NLC layer by applying an external voltage through the layer and exploiting the walk-off dependence on the beams power in the strongly nonlinear regime. ${ }^{11}$

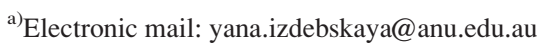

The external voltage is commonly used for beam steering in NLCs but involves application of inflexible electrode configurations at boundaries of a thin cell limiting the spatial variation of the molecular director and restricting the routing of light to the mid-plane of the cell. ${ }^{8}$ On the other hand, the externally applied magnetic field lacks those limitations and can be effectively used for the reorientation of optical axis of NLCs at will. Nevertheless, the first demonstration of the magnetic field control of the NR by Kang et al. ${ }^{10}$ was restricted to the prism geometry and was used only in the linear regime of free-propagating beams.

Recently, we have demonstrated the efficient magnetic field control of nematic liquid crystal orientation and nonlinear light-NLC interaction without the usual geometrical limitations. ${ }^{16}$ This approach provides an excellent condition for the spatial soliton formation and steering without any restriction on light trajectory.

In this letter, we report on controllable refraction of light beams at the interface between free space and positive uniaxial NLCs. In particular, we demonstrate the 3D-angular control of the refracted extraordinary polarized wave and the transition from positive to negative refraction for both freepropagating and solitons forming waves, by exploiting the light walk-off dependence on the orientation of the magnetic field.

Let us consider the experimental setup shown in Fig. 1. We use a cell with the nematic liquid crystal $6 \mathrm{CHBT}$, confined between two parallel glass slides $(10 \times 10 \mathrm{~mm})$ spaced by $1 \mathrm{~mm}$ along the $z$-axis. The inner surfaces of the slides were treated for homeotropic anchoring, to induce the initial alignment of the NLC molecules predominantly along the $z$-direction, i.e., perpendicularly to the glass surfaces. Due to the cell geometry, the NLC bulk was not restricted by the lateral boundary conditions along the radial coordinate $r$ parallel to the cell surfaces. An external static magnetic field from a permanent magnet was applied to provide the desired molecular reorientation in the NLC bulk. The corresponding orientation angle of molecules can be adjusted by varying the spatial orientation of the magnetic field. A circularly polarized monochromatic Gaussian beam traveling in air with a wavelength $\lambda$ falls on the input glass slide of the cell. If the $z$-axis is chosen to be orthogonal to the input interface, 


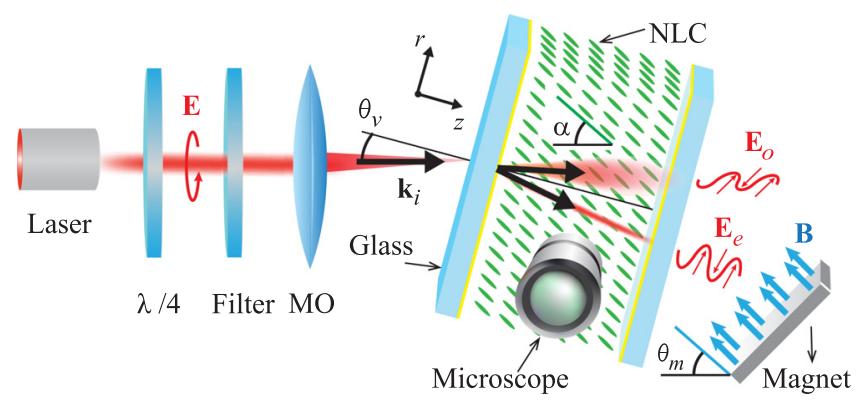

FIG. 1. Schematic of the set-up geometry used in negative refraction experiments. The input linearly polarized beam is converted into a circularly polarized beam and after transmission filter is focused with $0.45 \mathrm{NA}(20 \times)$ microscope objective (MO) on the glass-NLC interface. Inside the liquid crystal cell, the beam splits into two ordinary $(o)$ and extraordinary $(e)$ beams. The double-refraction angles are controlled by varying molecular reorientation of liquid crystal molecules by the external static magnetic field provided by the magnet.

then $\theta_{v}$ is the incident angle. Since the air and glass slide are isotropic media with refractive indices $n_{a} \cong 1$ and $n_{i} \cong 1.5$, respectively, then, following the Snell-Descartes law, the beam changes its direction at an angle $\theta_{i}=\arcsin \left(n_{i} \sin \theta_{v}\right)$ with respect to the $z$-axis in the plane of incidence $r z$. Subsequently, this tilted beam with wavevector $\mathbf{k}_{i}$ and the Poynting vector $\mathbf{S}_{i}$ encounters a planar interface at $z=0$ separating isotropic and positive uniaxial NLC media.

Fig. 2 illustrates what happens when the beam crosses such interface and the refracted wave becomes a subject to double refraction with wavevectors $\mathbf{k}_{o}$ for ordinary and $\mathbf{k}_{e}$ for extraordinary polarized components. The half-space $z>0$ with the NLC is macroscopically described by an angular orientation $\alpha$ of the average molecular director $\mathbf{n}$ with respect to $z$ and by refractive indices $n_{\|}$and $n_{\perp}\left(n_{\|}>n_{\perp}\right)$ for electric fields of light parallel or perpendicular to the molecular director $\mathbf{n}$, respectively. If $\theta_{t}$ is an angle between the $z$-axis and wavevector $\mathbf{k}_{e}$, then the refractive index is $n_{o}=n_{\perp}$ for ordinary and $n_{e}^{2}=\left(\left[\cos \left(\theta_{t}+\alpha\right) / n_{\perp}\right]^{2}+\left[\sin \left(\theta_{t}+\alpha\right) / n_{\|}\right]^{2}\right)^{-1}$, for extraordinary waves, respectively. The Poynting vector $\mathbf{S}_{\mathbf{o}}$ of the $o$-wave lies in the plane of incidence $r z$ at an angle $\theta_{o}=\angle \mathbf{k}_{o}, \mathbf{z}$, while the Poynting vector $\mathbf{S}_{\mathbf{e}}$ associated with the

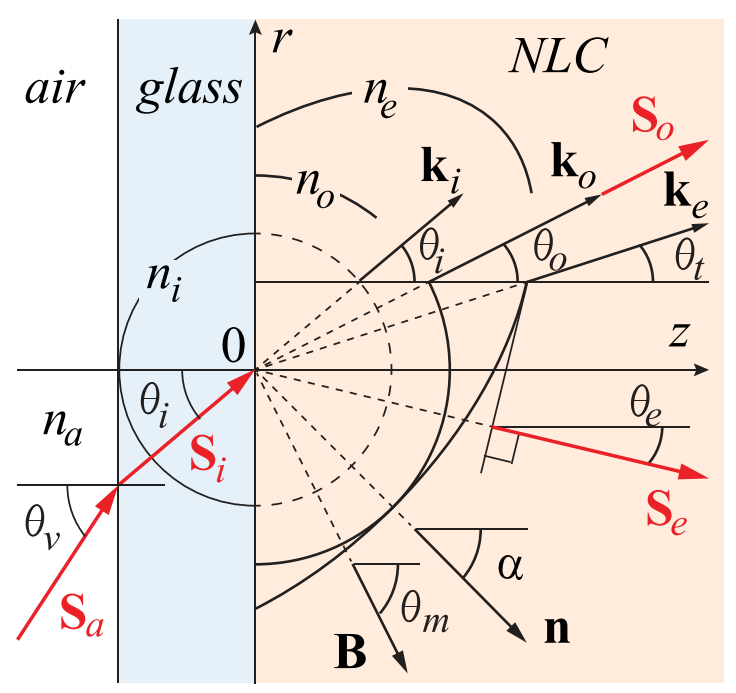

FIG. 2. Refraction of the ordinary and extraordinary waves at the isotropicNLC interface in the presence of the magnetic field. $e$-wave lies in the extraordinary principal plane $k_{e} n=z n$ forming an angle $\theta_{e}$ with respect to $z$.

In our geometry, the angular orientation $\alpha$ of director $\mathbf{n}$ is a result of applying a stationary homogeneous magnetic field $\mathbf{B}$ at an angle $\theta_{m}$ with respect to $z$. Therefore, $\alpha=0$ in the absence of the magnetic field and $\alpha=\theta_{m}$ when the magnetic field is strong enough to completely reorient the molecular director. In the latter case, which is the main focus of this work, the director can be precisely controlled by a manipulating angular position of the magnet. For simplicity, we chose the magnetic field vectors to be parallel to the plane of incidence $r z$, and thereafter, this plane is coplanar with the principal plane $z n$.

There are three different regimes for the $e$-wave propagation in NLCs, depending on the input light intensity and its ability to realign local molecular director: the linear, the perturbative nonlinear, and the strongly nonlinear regimes, respectively. ${ }^{17,18}$ Here, we consider the linear and perturbative nonlinear regimes ${ }^{17}$ for which the walk-off of an extraordinary beam is independent of the beam intensity and is defined only by the background molecular orientation. The examples of behaviour of ordinary and extraordinary beams in these regimes are shown in Fig. 3. The ordinary beam experiences only positive refraction independently of the orientation of the optical axis of the NLC defined by the external magnetic field. On the other hand, the extraordinary beam can experience either positive or negative refraction depending on the relationship between the incidence angle and the orientation of the magnetic field.

The linear regime of light propagation corresponds to a very low optical power, such that the light does not affect the orientation of director $\mathbf{n}$ and the extraordinary beam diffracts as it propagates [Figs. 3(a)-3(c)]. The perturbative nonlinear regime occurs when the electric field of the beam exerts torque on liquid crystal molecules rotating them by an angle $\psi$ such that $\theta_{e}=\alpha+\psi$ in the light beam intensity maxima. This yields a local increase of the extraordinary refractive index $n_{e}: \Delta n_{e}^{2}=n_{e}^{2}(\alpha)-n_{e}^{2}\left(\theta_{e}\right)$, leading to self-focusing of the beam [Figs. 3(e)-3(g)]. Importantly, in this perturbative nonlinear regime, the light-induced reorientation angle $\psi$ is sufficiently large to induce the self-focusing but still small
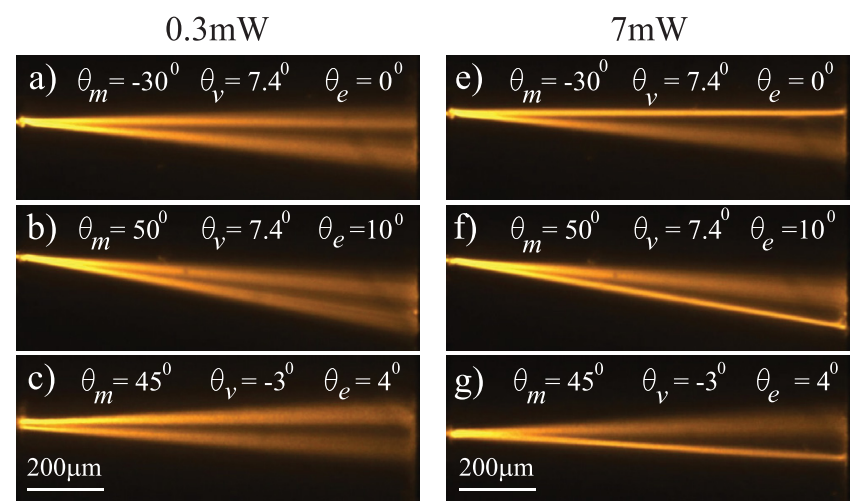

FIG. 3. Examples of double refraction in NLC in linear (a)-(c) and perturbative nonlinear $(\mathrm{e})-(\mathrm{g})$ regimes with power of incident circularly polarized beam $0.3 \mathrm{~mW}$ and $7 \mathrm{~mW}$, respectively. (a) and (e) show no refraction of the extraordinary $e$-wave (upper beam); (b) and (f) show positive refraction of the $e$-wave (lower beam); and (c) and (g) show negative refraction of the $e$ wave (lower beam). 

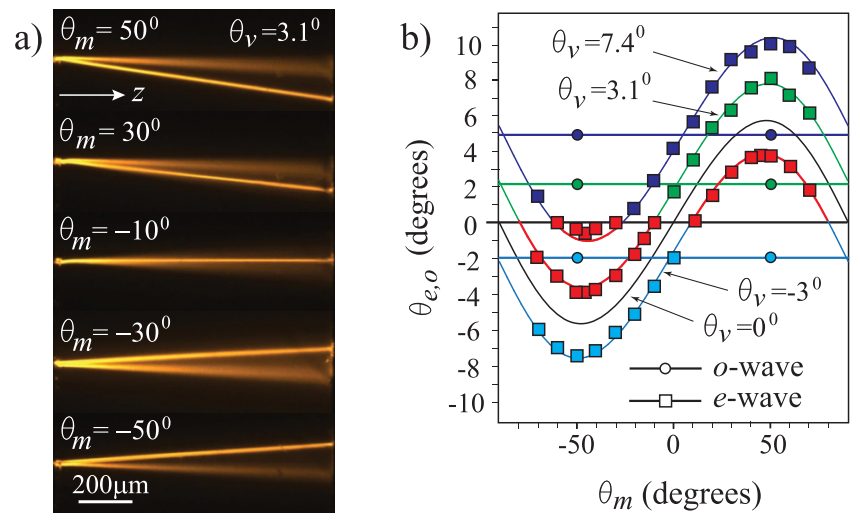

FIG. 4. Effect of the external magnetic field on light refraction in NLC. (a) Controllable switching between positive and negative refraction of the $e$-polarized spatial soliton formed at the input power $20 \mathrm{~mW}$. Here, the angle of incidence $\theta_{v}$ is fixed as $3.1^{\circ}$ and the angle $\theta_{m}$ of the magnetic field ranges from $-70^{\circ}$ to $70^{\circ}$. (b) Refraction angles of $o$-wave, $\theta_{o}$, and $e$-wave, $\theta_{e}$, vs. the angle $\theta_{m}$ of the magnetic field orientation. The experimental points are marked with squares for the $e$-wave and circles for the $o$-wave; the solid lines represent theoretical results obtained from Eq. (1) (curves) and from Eq. (2) (straight lines). The red squares correspond to negative refraction of the $e$-wave. Different colours correspond to different incidence angles.

with respect to $\alpha$ so it does not affect the Poynting vector's $\mathbf{S}_{\mathbf{e}}$ orientation $\theta_{e}$. In both linear and perturbation selffocusing regimes, the angle $\theta_{e}$ of the $e$-wave direction can be found as 7,8

$$
\theta_{e}=\arctan \frac{2 n_{i} \sin \theta_{i}-a n_{\perp} n_{\|} \sqrt{b n_{\perp}^{2} n_{\|}^{2}-n_{i}^{2} \sin ^{2} \theta_{i}}}{2 b n_{\perp} n_{\|} \sqrt{b n_{\perp}^{2} n_{\|}^{2}-n_{i}^{2} \sin ^{2} \theta_{i}}},
$$

where $a=\sin 2 \alpha\left(n_{\|}^{-2}-n_{\perp}^{-2}\right), b=n_{\perp}^{-2} \cos ^{2} \alpha+n_{\|}^{-2} \sin ^{2} \alpha$, and $\alpha \approx \theta_{m}$ for a strong magnetic field. The Poynting vector $\mathbf{S}_{o}$ of the $o$-wave propagates at the angle $\theta_{o}$ defined from SnellDescartes law:

$$
\theta_{o}=\arcsin \left(n_{i} \sin \theta_{i} / n_{o}\right)
$$

and is insensitive to both the intensity of the light beam and direction of the static magnetic field. In the above geometry, when the sign of the angle $\theta_{e}\left(\theta_{i}, \theta_{m}\right)$ is opposite to $\theta_{o}$, the $e$-wave undergoes negative refraction, as pictured in Fig. 2.

Figure 4 shows the experimentally observed cases of positive and negative refractions of the $e$-wave in both linear and nonlinear self-guiding perturbation regimes. In the experiment, a circularly polarized beam $(\lambda=800 \mathrm{~nm})$ was focused onto the glass-NLC interface of the cell filled with commercial liquid crystal 6CHBT $\left(n_{\perp}=1.5144\right.$ and $n_{\|}$ $=1.6714)$. The power of the beam can be smoothly varied by the rotating transmission filter from $0.1 \mathrm{~mW}$, where the intensity is well below the optically induced torques on $\mathbf{n}$, and the light propagation regime is purely linear, up to 20 $\mathrm{mW}$ - the power high enough for the self-focusing due to reorientational response leading to the formation of spatial solitons, the so-called nematicons. ${ }^{19,20}$ The images depict trajectories of light beams and solitons in the plane $r z$, acquired from a side-view microscope (MO) and a CCD camera. To control the refraction, we varied both an angle of incidence beam $\theta_{v}$ by tilting the cell and the magnetic field orientation by changing an angle $\theta_{m}$ in the plane of incidence $r z$. We used a cylindrical permanent magnet (diameter $20 \mathrm{~mm}$ ) placed $10 \mathrm{~mm}$ from the entrance point of the beam with the field strength $B=0.2 \mathrm{~T}$ at that point. The magnet orientation could be varied in the $-90^{\circ}<\theta_{m}<+90^{\circ}$ range.

The reorientational effect of an external magnetic field on the refraction phenomenon and transition from positive to negative refraction is illustrated in Figure 4. As it is clear from the graph in Fig. 4(b), there is a range of angles of incidence $\theta_{v}$ together with an angle of director orientation $\alpha=\theta_{m}$, for which the refraction is negative, i.e., the input and refracted beams appear on the same side of the normal to the interface. The maximal angle of negative refraction $\theta_{e}^{\max }= \pm \arctan \left[\left(n_{\|}^{2}-n_{\perp}^{2}\right) / 2 n_{\perp} n_{\|}\right]$is achieved when $\theta_{v} \rightarrow 0$ and $\theta_{m} \rightarrow \arctan \left(n_{\|} / n_{\perp}\right)$.

To conclude, we utilised sample geometry with magnetically controlled director in NLCs, which allows precision control of beam refraction, including negative refraction, in both linear and nonlinear regimes. Such controllable refraction of light via manipulation of the director of molecular orientation in the bulk NLC can be useful in a variety of light steering devices based on refraction phenomenon.

The work was supported by the Australian Research Council and the Qatar National Research Fund (Grant No. NPRP 9-020-1-006).

${ }^{1}$ A. D. Boardman, N. King, and L. Velasco, "Negative refraction in perspective," Electromagnetics 25, 365-389 (2005).

${ }^{2}$ V. G. Veselago, Sov. Phys. Usp. 10, 509-514 (1968).

${ }^{3}$ N. I. Zheludev, Science 328, 582-583 (2010).

${ }^{4}$ Y. Zhang, B. Fluegel, and A. Mascarenhas, Phys. Rev. Lett. 91, 157404 (2003).

${ }^{5}$ X. L. Chen, M. He, Y. X. Du, W. Y. Wang, and D. F. Zhang, Phys. Rev. 72, 113111 (2005).

${ }^{6}$ Z. Liu, Z. Lin, and S. T. Chui, Phys. Rev. B 69, 115402 (2004).

${ }^{7}$ H. Luo, W. Hu, X. Yi, H. Liu, and J. Zhu, Opt. Commun. 254, 353 (2005).

${ }^{8}$ O. P. Pishnyak and O. D. Lavrentovich, Appl. Phys. Lett. 89, 251103 (2006).

${ }^{9}$ M. Peccianti and G. Assanto, Opt. Express 15, 8021 (2007).

${ }^{10}$ L. Kang, Q. Zhao, B. Li, J. Zhou, and H. Zhu, Appl. Phys. Lett. 90, 181931 (2007).

${ }^{11}$ A. Piccardi, A. Alberucci, N. Kravets, O. Buchnev, and G. Assanto, Nat. Commun. 5, 5533 (2014).

${ }^{12}$ H. Harutyunyan, R. Beams, and L. Novotny, Nat. Phys. 9, 423 (2013).

${ }^{13}$ C. Luo, S. G. Johnson, J. D. Joannopoulos, and J. B. Pendry, Phys. Rev. B 65, 201104 (2002).

${ }^{14}$ E. Cubukcu, K. Aydin, E. Ozbay, S. Foteinopoulou, and C. Soukoulis, Nature 423, 604 (2003).

${ }^{15}$ P. G. de Gennes and J. Prost, The Physics of Liquid Crystals (Oxford Science, 1993).

${ }^{16}$ Y. Izdebskaya, V. Shvedov, G. Assanto, and W. Krolikowski, Nat. Commun. 8, 14452 (2017).

${ }^{17}$ M. Peccianti, C. Conti, G. Assanto, A. De Luca, and C. Umeton, Nature 432, 733 (2004).

${ }^{18}$ A. Piccardi, G. Assanto, L. Lucchetti, and F. Simoni, Appl. Phys. Lett. 93, 171104 (2008).

${ }^{19}$ M. Peccianti and G. Assanto, Phys. Rep. 516, 147 (2012).

${ }^{20}$ G. Assanto, Nematicons: Spatial Optical Solitons in Nematic Liquid Crystals (John Wiley and Sons, New York, 2012). 